\title{
Self-Reported Health Problems of Young Adults in Clinical Settings: Survivors of Childhood Cancer and Healthy Controls
}

\author{
Lisa A. Schwartz, PhD, Jun J. Mao, MD, MSCE, Branlyn W. DeRosa, PhD, \\ Jill P. Ginsberg, MD, Wendy L. Hobbie, MSN, CRNP, Claire A. Carlson, BSN, RN, \\ Ifigenia D. Mougianis, BA, Sue K. Ogle, MSN, CRNP, and Anne E. Kazak, PhD
}

Purpose: Increasing numbers of childhood cancer survivors are being seen in primary care settings as young adults. It is unknown how their self-reported health problems differ from those of healthy young adults. Self-reported health problems of cancer survivors and healthy controls are compared in this study.

Methods: 156 cancer survivors visiting a cancer survivorship program and 138 controls in primary care centers (mean age, 20 years) completed the Health Knowledge Inventory, a checklist of 35 health problems.

Results: Cancer survivors reported significantly more health problems than healthy controls $(5.6 \mathrm{vs}$ 2.6 problems; $P<.001)$. For cancer survivors, more intense treatment and older age related to Organic/Major problems and Constitutional/0ther problems. Female sex related to report of Organic/Major problems and Constitutional/Other problems for the controls. Although at least $20 \%$ of both healthy controls and survivors endorsed dermatologic, headache, gastrointestinal, and weight problems, survivors endorsed growth, thyroid, kidney, immunologic, heart, and fertility problems 4-fold over controls.

Conclusions: Cancer survivors endorse significantly more health problems than do healthy controls. However, some problems are reported with equal frequency among the groups. Understanding these similarities and differences between survivors and healthy controls will facilitate patient-centered comprehensive care for young-adult cancer survivors. (J Am Board Fam Med 2010;23:306-314.)

Keywords: Checklist, Young Adult, Primary Health Care, Child, Cancer, Cancer Survivorship

Given significant treatment advances in recent decades, the survival rate for pediatric cancer exceeds $75 \%$ and 1 out of every 640 young adults is a pediatric cancer survivor. ${ }^{1,2}$ Despite being cured, childhood cancer survivors experience or are at risk for sequelae of their disease or treatment (ie, late effects) that often do not appear until adulthood. ${ }^{3}$

This article was externally peer reviewed.

Submitted 8 September 2009; revised 13 November 2009; accepted 10 December 2009.

From the Division of Oncology, The Children's Hospital of Philadelphia, Pennsylvania (LAS, BWD, JPG, WLH, CAC, IDM, SKO, AEK); the Department of Family Medicine and Community Health (JJM) and the Department of Pediatrics (JPG, AEK), University of Pennsylvania School of Medicine, Philadelphia.

Funding: Funding was provided by the National Cancer Institute (R01 CA106928) to AEK.

Conflict of interest: none declared.

Corresponding author: Lisa A. Schwartz, PhD, Division of Oncology, The Children's Hospital of Philadelphia, 34th and Civic Center Boulevard, CHOP North \#1484, Philadelphia, PA 19104 (E-mail: schwartzl@email.chop.edu).
Late effects may be medical (eg, cardiovascular, pulmonary, renal, musculoskeletal, endocrinopathies, second cancers) or psychological (eg, cognitive, depression, posttraumatic stress). ${ }^{4}$ Life-long medical surveillance is necessary to manage these concerns, monitor risk for future morbidities of cancer therapy, and provide counseling about health risk and promotion behaviors to help reduce the occurrence or severity of late effects. ${ }^{2,5}$

Unfortunately, data from the Childhood Cancer Survivorship Study (CCSS) has shown that only $35 \%$ of survivors recognize that they could have serious health problems related to their cancer treatment ${ }^{6}$ and more than $50 \%$ do not receive cancer-related follow-up care. ${ }^{7}$ Family physicians tend to see more young adults than other physician specialties and may therefore play an important role in addressing the needs of cancer survivors in this age range. ${ }^{8,9}$ The potential benefits of involvement of a primary care physician (PCP) include continuity of 
care, geographic convenience, cost effectiveness, and treatment in settings that are developmentally appropriate (not pediatric) and void of negative reminders of the cancer experience. PCPs are also able to treat both cancer- and noncancer-related problems, which is especially important given predictions that the number of oncologists is insufficient to meet the needs of the increasing numbers of cancer survivors. ${ }^{9}{ }^{10-12}$ To provide optimal survivorship care, PCPs need to be knowledgeable about the health problems of cancer survivors and how survivors present their concerns relative to healthy young adults. ${ }^{11}$

Although previous epidemiologic research has demonstrated that adult survivors of childhood cancer report more health problems than a sibling control, ${ }^{13}$ how the health problems of cancer survivors compare with unrelated young adults without a history of a chronic health condition in clinical settings is not known. In fact, little is known about the presenting problems of young adults, in general, because they are underinsured and underutilize health care. ${ }^{14}$ When young adults do seek medical care it is more likely to be initiated for a suspected problem or illness rather than a routine visit. ${ }^{14}$ Thus, because of the limited research about the health concerns of young adults, an understanding of young adult self-reported health problems during a clinical encounter would allow PCPs to effectively and efficiently probe for problems that may be common for young adults, in general, and to young-adult cancer survivors, in particular. Therefore, the aim of this study was to compare report of health problems by young adults in survivorship care to those by young adults without significant medical histories seen in primary care. We also sought to identify the relationship between clinical and demographic variables and reported problems for each group separately.

\section{Methods}

\section{Study Design and Patient Population}

The study was part of a larger investigation of outcomes of young-adult cancer survivors compared with young adults without significant health histories. The Children's Hospital of Philadelphia and University of Pennsylvania institutional review boards approved the study. Participants attended an outpatient medical visit at the Children's Hospital of Philadelphia Cancer Survivorship Program
(CSP) or at a primary care office (controls). Patients in the CSP were seen by a pediatric oncologist or a nurse practitioner with expertise in cancer survivorship. Those attending a primary care visit were seen by a family medicine, adolescent medicine, or internal medicine physician. Eligibility criteria included an age of 16 to 29 years, Englishspeaking, 6th-grade reading level, cognitive capability to complete questionnaires, and availability of a parent to provide consent for those younger than age 18. Additional eligibility criteria for survivors included diagnosis of a childhood malignancy before age 21 , at least 5 years since diagnosis, and $\geq 2$ years since completion of cancer treatment. Brain tumor patients were excluded because of potential cognitive impairments. Young adults in the control condition were excluded if they were pregnant or had a history of a chronic health condition, life-threatening injury, or psychiatric condition requiring hospitalization.

Survivors were recruited consecutively during their yearly CSP visit. Healthy young adults were recruited during a primary care visit at University of Pennsylvania Health System practices. After consent and assent for those younger than age 18, participants completed self-report questionnaires. Controls were recruited in a targeted fashion to obtain similar demographic (ie, age, minority status, sex) distributions to the survivors using a stratification table. At the family and adolescent practices, potential participants were identified and screened for study criteria using the computerized medical record. All potential control participants, regardless of prescreening, were screened for eligibility in person by a research assistant using a structured checklist of medical problems.

\section{Measures}

Self-Reported Health Problems

The Health Knowledge Inventory (HKI), the primary outcome measure used in this study, was developed by a team of survivorship experts to measure health problems. It has 3 parts: Part 1, Knowledge of Disease and Treatment; Part 2, Perceptions of Treatment and Late Effect Severity; and Part 3, Current Medical Problems (35 categories of problems plus an "other" category). Only Part 3 was used in this report, and a version for controls contains only Part 3. Clinical expertise (from both internal and external survivorship and family medicine providers) and literature reviews of 
late effects were used to develop the 35 items (health problems) and to categorize symptoms into 2 categories: Organic/Major (ie, related to major organ system and/or a substantial late effect of childhood cancer) or Constitutional/Other (ie, a problem related to the whole system, and therefore may not be specific, such as fatigue, or a problem deemed less medically substantial and threatening such as taste or hearing problems). Although they are inclusive of all potential childhood cancer late effects, the problem list represents a general list of symptoms that may be applicable to any individual, irrespective of cancer history. Patients were provided with a list of health problems with examples (eg, heart/blood problems: weak heart, chest pain, irregular heartbeat, high blood pressure) and asked to endorse (yes/no) whether or not they have each of the 35 potential categories of problems. A total summary score was calculated from positive endorsements of problems (maximum of 35). Individual summary scores were also calculated separately for Organic/Major problems and Constitutional/ Other problems.

\section{Covariates}

Age, ethnicity/race, highest education attained, and personal income were dichotomized to age 16 to 19/20 and above (median age, 20); non-Hispanic white/minority; completed high school or less/pursued education beyond high school; and personal income less than or more than $\$ 20,000$. For the cancer survivors, diagnosis, stage, and treatment modality (radiation, chemotherapy, surgery, stem cell transplantation) was extracted from chart review to calculate treatment intensity using the 4 levels of the Intensity of Treatment Rating scale. ${ }^{15}$ Rater reliability in prior studies was excellent. ${ }^{15,16}$

\section{Data Analysis}

Descriptive statistics were run on each variable. $\chi^{2}$ and $t$ test analyses were used to compare the groups on demographics and the number of reported health problems. For problems reported in at least $5 \%$ of both the control and survivor samples, logistic regression was used to assess the relationship of group (survivor/control) to report of each problem on part 3 of the HKI. Fisher's exact test was used to compare groups on reported problems when $<5 \%$ of participants reported the problem. Analyses of variance and $t$ tests were used to test the relationships among the clinical and demographic variables and report of Organic/Major and Constitutional/Other problems. Multivariate linear regression models were used to determine the independent relationship of demographic and clinical variables to the number of reported problems for each group. In particular, significant associates $(P<.05)$ of a report of Organic/Major problems were used in a model testing Organic/Major problems. Similarly, significant associates $(P<.05)$ of a report of Constitutional/Other problems were used in a model testing Constitutional/Other problems.

\section{Results}

\section{Participant Characteristics}

Of the 211 cancer survivors who were approached, 180 consented (85.3\% participation rate) and 156 (73.9\%) completed the measures. Cancer diagnoses for the survivors were leukemias (44.9\%), lymphomas $(19.2 \%)$, and solid tumors $(35.9 \%)$. On the Intensity of Treatment Rating, ratings of treatment intensity were: least severe, $2.7 \%$; moderately severe, $42.7 \%$; very severe, $38.7 \%$; and most severe, $16 \%$. Because only 4 patients $(2.7 \%)$ were categorized as least severe, the lowest 2 categories (least and moderately severe) were combined for analysis. Median age at diagnosis was 7.71 years (range, 0-20 years). Median time since treatment was 9.87 years (range, 3.00-22.39 years). Most of the cancer survivors were non-Hispanic white (86.5\%); 6.4\% were African American, 3.2\% were Asian, 2.6\% were Hispanic, and $1.3 \%$ unknown.

For the control group, 238 were approached, 181 consented (76.1\% participation rate), and 138 completed the study and matched the demographics of the survivorship group. (A stratification sampling strategy, the aim of which is to attain a final control group with demographics similar to the cancer survivor group, is being used in the ongoing study. Because data collection is ongoing, only control group participants with characteristics matching those of the current survivor sample were used). Reasons for attending a primary care visit were routine visit $(45.5 \%)$, follow-up visit $(23.9 \%)$, or acute problem (30.6\%). Most of the healthy controls were non-Hispanic white $(81.9 \%)$, whereas the remaining were African American (11.6\%), Asian (3.6\%), Hispanic (0.7\%), and more than one race $(2.2 \%)$. The groups did not differ in age, sex, minority status, education level, or personal income (Table 1). 


\begin{tabular}{lcrr}
\hline & Survivors $(\mathrm{n}=156)$ & Controls (n = 138) & $P$ \\
\hline Age (mean [SD]) & $19.9(3.1)$ & $20.6(2.9)$ & .06 \\
Male gender (n [\%]) & $75(48)$ & $60(44)$ & .43 \\
Non-Hispanic white (n [\%]) & $135(87)$ & $113(82)$ & .27 \\
Education beyond high school (n [\%]) & $83(54)$ & $79(58)$ & .44 \\
Personal income $<\$ 20,000(\mathrm{n}[\%])$ & $115(79)$ & $96(72)$ & .14 \\
\hline
\end{tabular}

\section{Self-Report of Health Problems}

Cancer survivors reported significantly more health problems based on the total score on the HKI than controls (5.6 vs 2.6; $P<.001$; Table 2). They also reported significantly more Organic/Major problems (2.9 vs $1.3 ; P<.001)$ and Constitutional/ Other problems $(2.7$ vs $1.4 ; P<.001)$ on the HKI subscales (Table 2). For the majority of problems on the HKI (31 of 35), more survivors endorsed the problem than did controls (Table 3). Problems common among both groups (ie, $\geq 20 \%$ endorsement) were dermatologic, headache, gastrointestinal, and weight problems. However, survivors endorsed problems related to growth, hearing, thyroid, kidney, liver, immunologic, heart, and fertility almost 4 times more than did the healthy controls. For the problems that occurred in at least $5 \%$ of the sample, odds ratios ranged from .8 (bladder/urination) to 4.4 (vision/eye problems) for the Organic/Major problems and .7 (other pain) to 12.2 (significant scars) among the Constitutional/ Other problems.

An examination of factors associated with selfreport of problems was conducted for each group separately (Table 4). Significant correlates of selfreported problems on the HKI served as independent variables in multivariate regression models predicting report of problems on the HKI by group (Table 5). For survivors, older age and more intensive cancer treatment predicted Organic/Major problems $(P<.001)$. Older age, higher treatment intensity, and having a solid tumor predicted Con-
stitutional/Other problems for the survivors $(P<$ .001). For the controls, regression analyses showed that female sex and being white were significant predictors of Organic/Major problems $(P<.05)$. Female sex was the only significant correlate of Constitutional/Other problems for the control group $(P<.001)$; thus, multivariate regression analysis was not conducted for this outcome for controls.

\section{Discussion}

To our knowledge, this is the first study comparing self-reported health problems of young-adult cancer survivors and young adults without a history of a chronic health condition during a medical visit. Our findings are consistent with those from the CCSS in that cancer survivors report significantly more health problems compared with controls. ${ }^{13}$ Although the results of the CCSS help define the incidence and prevalence of late effects among survivors through epidemiologic survey methodologies, our study provides additional clinically useful information about how survivors may differ from individuals of similar age who present during a medical visit. In addition, the present study used unrelated controls compared with the sibling controls used in the CCSS-individuals that may not represent true "healthy" and unaffected individuals. Specifically, siblings of childhood cancer survivors often experience psychological distress and engage in health-hurting behaviors that may compromise their health and well-being. ${ }^{17,18}$

Table 2. Self-Report of Health Problems on the Health Knowledge Inventory by Group

\begin{tabular}{lccr}
\hline Health Knowledge Inventory Problems & Survivors $(\mathrm{n}=156)$ & Controls $(\mathrm{n}=138)$ & $P$ \\
\hline Total & $5.6(4.0)$ & $2.6(2.8)$ & 0.00 \\
Organic/major & $2.9(2.3)$ & $1.3(1.6)$ & 0.00 \\
Constitutional/other & $2.7(2.1)$ & $1.4(1.6)$ & 0.00 \\
\hline
\end{tabular}

Values provided as mean (SD). 
Table 3. Comparison of Health Knowledge Inventory Problems Endorsed by Group

\begin{tabular}{|c|c|c|c|}
\hline Problems & Survivors* $(\mathrm{n}=156)$ & Controls* $(\mathrm{n}=138)$ & Odds Ratio $(95 \% \mathrm{CI})^{\dagger}$ \\
\hline \multicolumn{4}{|l|}{ Organic/major } \\
\hline Growth & $32(20.5)$ & $1(0.7)$ & \\
\hline Seizure & $1(0.6)$ & $1(0.7)$ & \\
\hline Stroke & $0(0.0)$ & $0(0.0)$ & \\
\hline Hearing & $21(13.9)$ & $5(3.6)$ & \\
\hline Thyroid & $14(9.0)$ & $1(0.7)$ & \\
\hline Heart/blood & $27(17.3)$ & $5(3.6)$ & \\
\hline Breast & $10(6.4)$ & $3(2.2)$ & \\
\hline Kidney & $13(8.3)$ & $3(2.2)$ & \\
\hline Liver & $6(3.8)$ & $1(0.7)$ & \\
\hline Diabetes-related & $1(0.6)$ & $2(1.4)$ & \\
\hline Immunologic & $8(5.1)$ & $0(0.0)$ & \\
\hline Other endocrine & $6(3.8)$ & $1(0.7)$ & \\
\hline Second cancer & $5(3.2)$ & $0(0.0)$ & \\
\hline Vision/eye & $49(31.4)$ & $13(9.4)$ & $4.4(2.3-8.6)$ \\
\hline Reproductive & $38(24.4)$ & $10(7.2)$ & $4.1(2.0-8.6)$ \\
\hline Bone/joint & $43(27.6)$ & $14(10.1)$ & $3.4(1.8-6.5)$ \\
\hline Breathing/lung & $28(17.9)$ & $12(8.7)$ & $2.3(1.1-4.7)$ \\
\hline Cognitive/learning & $30(19.2)$ & $16(11.6)$ & $1.8(0.9-3.5)$ \\
\hline Dermatologic & $41(26.3)$ & $32(23.2)$ & $1.2(0.7-2.0)$ \\
\hline Stomach/gastrointestinal & $33(21.2)$ & $29(21.0)$ & $1.0(0.6-1.8)$ \\
\hline Bladder/urination & $11(7.1)$ & $12(8.7)$ & $0.8(0.3-1.9)$ \\
\hline \multicolumn{4}{|l|}{ Constitutional/other } \\
\hline Face looks different & $11(7.1)$ & $0(0.0)$ & \\
\hline Body looks different & $30(19.2)$ & $3(2.2)$ & \\
\hline Mouth/taste problems & $17(10.9)$ & $4(2.9)$ & \\
\hline Problems walking & $5(3.2)$ & $2(1.4)$ & \\
\hline Balance problems & $12(7.7)$ & $4(2.9)$ & \\
\hline Significant scars & $101(64.7)$ & $18(13.0)$ & $12.2(6.8-22.2)$ \\
\hline Getting tired easily & $43(27.6)$ & $18(13.0)$ & $2.5(1.4-4.7)$ \\
\hline Other & $5(13.9)$ & $7(6.3)$ & $2.4(0.7-8.2)$ \\
\hline Dental/gum & $28(17.9)$ & $13(9.4)$ & $2.1(1.0-4.3)$ \\
\hline Psychologic & $41(26.3)$ & $23(16.7)$ & $1.8(1.0-3.2)$ \\
\hline Sleep & $36(23.1)$ & $25(18.1)$ & $1.4(0.8-2.4)$ \\
\hline Headaches & $72(40.0)$ & $41(29.7)$ & $1.4(0.9-2.3)$ \\
\hline Weight & $39(25.0)$ & $27(19.6)$ & $1.4(0.8-2.4)$ \\
\hline Nose/smell & $16(10.3)$ & $11(8.0)$ & $1.3(0.6-3.0)$ \\
\hline Other pain & $12(7.7)$ & $14(10.1)$ & $0.7(0.3-1.7)$ \\
\hline
\end{tabular}

*Values in these columns provided as $\mathrm{n}(\%)$.

${ }^{\dagger}$ Odds ratio and CIs reported only for health problems endorsed by at least $5 \%$ of both groups. Two-tailed Fisher's exact test was run for health problems with $<5 \%$ frequency of endorsement by either group.

Young adult cancer survivors self-reported more than twice the number of health problems as young adults without a history of a chronic health condition. Survivors were more likely to report problems that may require significant medical intervention or monitoring such as cardiac, pulmonary, reproductive, vision/eye, thyroid, kidney, growth, and bone/joint health is- sues. However, both groups tended to report problems exacerbated by stress and that may relate to both physical and psychological difficulties. Furthermore, female sex was associated with the report of health problems among the healthy young adults but not among cancer survivors.

Despite the increased frequency of significant problems reported by survivors, some problems 
Table 4. Comparison of Number of Self-Reported Organic/Major and Constitutional/Other Problems among Survivors and Controls by Clinical and Demographic Categories

\begin{tabular}{|c|c|c|c|c|c|c|c|c|}
\hline & \multicolumn{4}{|c|}{ Survivors $(\mathrm{n}=156)$} & \multicolumn{4}{|c|}{ Controls $(\mathrm{n}=138)$} \\
\hline & $\begin{array}{l}\text { Organic/ } \\
\text { Major }\end{array}$ & $P^{*}$ & $\begin{array}{c}\text { Constitutional/ } \\
\text { Other }\end{array}$ & $P^{*}$ & $\begin{array}{l}\text { Organic/ } \\
\text { Major }\end{array}$ & $P^{\star}$ & $\begin{array}{c}\text { Constitutional/ } \\
\text { Other }\end{array}$ & $P^{*}$ \\
\hline Diagnosis $^{\dagger}$ & & .10 & & .02 & & & & \\
\hline Leukemia & $2.64(2.37)$ & & $2.23(1.97)$ & & N/A & & N/A & \\
\hline Lymphoma & $2.40(1.87)$ & & $2.70(2.38)$ & & N/A & & N/A & \\
\hline Solid tumor & $3.36(2.30)$ & & $3.30(2.02)$ & & N/A & & N/A & \\
\hline Intensity of Treatment Rating $2^{\ddagger}$ & & .00 & & .02 & & & & \\
\hline Least/moderate & $1.93(1.65)$ & & $2.09(2.19)$ & & N/A & & N/A & \\
\hline Very & $3.33(2.30)$ & & $3.07(1.93)$ & & N/A & & N/A & \\
\hline Most & $4.50(2.65)$ & & $3.38(1.93)$ & & N/A & & N/A & \\
\hline Reason for visit & & & & & & .62 & & .79 \\
\hline Routine & N/A & & N/A & & $1.26(1.67)$ & & $1.46(1.59)$ & \\
\hline Follow-up & N/A & & N/A & & $1.44(1.68)$ & & $1.22(1.84)$ & \\
\hline Acute & N/A & & N/A & & $1.07(1.37)$ & & $1.37(1.48)$ & \\
\hline Age (years) & & .03 & & .03 & & .43 & & .39 \\
\hline$<20$ & $2.46(2.21)$ & & $2.35(1.93)$ & & $1.40(1.77)$ & & $1.53(1.77)$ & \\
\hline$\geq 20$ & $3.24(2.29)$ & & $3.06(2.24)$ & & $1.18(1.46)$ & & $1.28(1.51)$ & \\
\hline Gender & & .02 & & .15 & & .00 & & .00 \\
\hline Male & $2.43(2.23)$ & & $2.45(1.88)$ & & $0.78(1.12)$ & & $0.85(1.26)$ & \\
\hline Female & $3.25(2.26)$ & & $2.94(2.29)$ & & $1.63(1.78)$ & & $1.78(1.74)$ & \\
\hline Ethnicity/race & & .69 & & .90 & & .00 & & .19 \\
\hline White & $2.88(2.27)$ & & $2.70(2.12)$ & & $1.43(1.67)$ & & $1.46(1.64)$ & \\
\hline Minority & $2.67(2.37)$ & & $2.76(2.10)$ & & $0.48(0.71)$ & & $1.00(1.47)$ & \\
\hline Highest education & & .14 & & .05 & & .57 & & 1.00 \\
\hline$\leq 12$ th grade & $2.58(2.46)$ & & $2.36(2.14)$ & & $1.14(1.37)$ & & $1.37(1.62)$ & \\
\hline$>12$ th grade & $3.12(2.08)$ & & $3.02(2.06)$ & & $1.29(1.61)$ & & $1.37(1.60)$ & \\
\hline Income & & .14 & & .19 & & .87 & & .50 \\
\hline$<\$ 20,000$ & $2.71(2.25)$ & & $2.62(2.03)$ & & $1.24(1.53)$ & & $1.42(1.74)$ & \\
\hline$\geq \$ 20,000$ & $3.40(2.18)$ & & $3.20(2.48)$ & & $1.29(1.71)$ & & $1.24(1.22)$ & \\
\hline
\end{tabular}

All values provided as mean (SD).

${ }^{*} P$ values are based on $t$ tests or analyses of variance testing for within group (survivors or controls) differences on number of problems reported in each clinical and demographic category. Because diagnoses and Intensity of Treatment Rating included 3 categories, post hoc analyses using the least squares differences test was used to identify which of the 3 categories significantly differed from one another.

${ }^{\dagger}$ Post hoc analyses comparing problem reported by diagnosis found that those with solid tumors reported significantly more Constitutional/Other problems than those with leukemia.

${ }^{\ddagger}$ Post hoc analyses comparing the 3 categories of treatment intensity revealed that all categories differed significantly from one another on reporting of Constitutional/Other and Organic/Major problems.

were reported relatively frequently across both groups, including dermatological, gastrointestinal, bone/joint, weight, sleeping, and psychological problems as well as headaches. The frequency of the reporting of these problems is consistent with previous research about problem endorsement of young adults and adult cancer survivors. ${ }^{19,20}$ These may be issues that are common to the developmental stage of this population and PCPs may be more familiar with their presentation. However, the eti- ology and nature of these health problems may differ among cancer survivors and controls.

It should also be noted that healthy young adults in general do not often seek medical care. ${ }^{14}$ Those who do seek medical care may be motivated by an acute illness, a required physical for work or school, or other health concerns. Understanding the health problems of survivors compared with healthy controls in the setting of a clinical visit is important to help sort out what issues are unique to the experi- 
Table 5. Multivariate Regressions Predicting SelfReport of Health Problems among Categories with More Than One Correlate

\begin{tabular}{|c|c|c|c|}
\hline & $\begin{array}{l}\text { Variance } \\
\text { Accounted } \\
\text { For }(\%)\end{array}$ & $\beta^{*}$ & $P$ \\
\hline \multicolumn{4}{|l|}{ Organic/major problems } \\
\hline \multicolumn{4}{|l|}{ Survivors $(\mathrm{n}=156)$} \\
\hline Total model & 22 & & .00 \\
\hline $\mathrm{Age}^{\dagger}$ & & 0.15 & .04 \\
\hline $\mathrm{Sex}^{\neq}$ & & 0.14 & .07 \\
\hline Treatment intensity $^{\dagger}$ & & 0.38 & .00 \\
\hline \multicolumn{4}{|l|}{ Controls $(\mathrm{n}=138)$} \\
\hline Total model & 9 & & .00 \\
\hline Minority status ${ }^{\ddagger}$ & & -0.12 & .03 \\
\hline $\mathrm{Sex}^{\ddagger}$ & & 0.18 & .00 \\
\hline \multicolumn{4}{|l|}{ Constitutional/other problems } \\
\hline \multicolumn{4}{|l|}{ Survivors $(\mathrm{n}=156)$} \\
\hline Step $1^{\S}$ & 11 & & .00 \\
\hline Age & & 0.21 & .01 \\
\hline Treatment intensity $^{\dagger}$ & & 0.25 & .00 \\
\hline Step $2^{\|}$ & 7 & & .00 \\
\hline Lymphoma & & 0.13 & .13 \\
\hline Solid tumor & & 0.27 & .00 \\
\hline
\end{tabular}

${ }^{*} \beta$ is standardized.

${ }^{\dagger}$ Age and treatment intensity are entered as continuous variables to facilitate easier interpretation and to maximize variance of these variables. Thus, positive $\beta$ values indicate that problems increase with higher age and higher treatment intensity.

${ }^{\ddagger}$ Dichotomous variables are coded as 0 and 1 . In particular, sex is coded as $0=$ male, $1=$ female and minority status is coded as $0=$ white and $1=$ minority. Thus, a positive $\beta$ value for sex indicates a positive relationship between female sex and problem reporting. The negative $\beta$ value for minority status indicates a positive relationship between being white and report of problems.

${ }^{\mathfrak{S}}$ Two steps were used in the regression predicting Constitutional/Other problems in order to determine the unique contribution of diagnosis given the multiple categories in this variable. "Dummy codes for diagnosis were used to reflect categories of diagnoses of lymphoma and solid tumor.

ence of cancer survivorship as compared with health problems common among young adults seeking medical care. Thus, given that young adulthood is often considered a disease-free period and has received relatively little attention in research, our study highlights the unique presentation of health issues among young adult cancer survivors compared with their peers.

Predictors of problem reporting varied between the 2 groups. Although women and non-Hispanic whites reported more problems among the controls, age and clinical characteristics were most related to a number of health problems reported by cancer survivors. Older age has been shown to relate to problems for survivors, given the increase in late effects with age. ${ }^{3}$ That disease characteristics, which may be unknown to PCPs, were most related to problem reporting emphasizes the need for providers to have mechanisms for accessing treatment summaries and to collaborate with referring oncologists to better coordinate transfer of care. ${ }^{9,11}$ PCPs who are aware that a survivors' treatment was particularly intensive can use this knowledge to elicit information about the presence of problems if they are not mentioned by the patient. Coordinated care between oncology providers and PCPs, including communication of disease and treatment history and future risk, is ideal and is desired by cancer survivors. ${ }^{9,21}$ However, it should be noted that the regression models in this study explained relatively little of the variance of selfreported health problems. Thus, there is a need to identify other factors that may contribute to patient report of problems.

Limitations of this study exist. First, the HKI was designed to assess potential late effects on survivors. Though comprehensive, the questionnaire may not include all health problems relevant to young adults. In addition, the survivors were seen in a specialized survivorship clinic and it is not known if their reports of problems would be similar in a primary care setting. However, knowledge of the description of health problems of survivors seen in a survivorship clinic may increase generalists' awareness of what problems to assess when assuming survivorship care of young adults. The overall sample was mostly non-Hispanic whites, consistent with the general childhood cancer survivorship population. ${ }^{22,23}$ Finally, the control group in this study is not representative of all patients in primary care given that they did not have significant medical histories.

Despite the limitations, this study offers valuable insight from a clinical perspective about how young adult survivors of childhood cancer differ from individuals of a similar age without a history of chronic illness. Although both groups present with similar issues (eg, skin, weight, and gastrointestinal issues), survivors are more likely to present with problems of significant medical concern, requiring further evaluation and monitoring. Indeed, research has shown that the health problems of childhood cancer survivors increase throughout adulthood rather than plateau. ${ }^{3}$ Specifically, 30 years 
from diagnosis, $73 \%$ of survivors will develop at least one chronic physical condition and $42 \%$ will develop a condition that is life-threatening or severely disabling or they will have died from a chronic condition. ${ }^{3}$ Thus, the likelihood of increasing health problems and risk of future problems among survivors underscores the need for childhood cancer survivors to transition to adult care for long-term follow-up.

As many young adults survivors "age out" of pediatric oncology settings, PCPs have a unique opportunity to provide high-quality and accessible follow-up care for this at-risk population. Understanding the unique health problems of young adult survivors is one step in attaining proficiency in caring for them. Given that PCPs are trained in risk assessment, risk modification, cancer surveillance, chronic disease management, care coordination, and patient education, they have the skills critical to addressing the needs of young-adult childhood cancer survivors. ${ }^{8}$ Furthermore, because of the continuity of care provided by PCPs, they are well suited to provide the longitudinal, proactive, and anticipatory care required for optimal survivorship care. $^{24}$

The authors thank the participants for their assistance in this research. We also thank Joseph Straton, MD, Richard Ittenbach, $\mathrm{PhD}$, and Mary T. Rourke, $\mathrm{PhD}$, for their contributions to this project; Anna T. Meadows, MD, and Jeffrey Silber, MD, for completing the HKI; Stephanie K. Bui, MD, Janice K. Hillman, MD, FACP, Evelyn Wiener, MD, Michele Demski, BSN, RN, and Maureen Reilly, BSN, RN, for assisting with recruitment and access to patients; and Andrew Gaffney, Emily Knudsen-Strong, Muhammad Monsour, Sonali Sanyal, Mary Caitlin St. Clair, James Wolf, and Mindy Yang for serving as research assistants for this study.

\section{References}

1. Ries LAG, Eisner MP, Kosary CL, et al (eds). SEER Cancer Statistics Review, 1973-1999. Bethesda, MD: National Cancer Institute; 2002.

2. Hewitt M, Weiner SL, Simone JV, eds. Childhood cancer survivorship: improving care and quality of life. Washington, DC: National Academies Press; 2003.

3. Oeffinger KC, Mertens AC, Sklar CA, et al. Chronic health conditions in adult survivors of childhood cancer. N Engl J Med 2006;355:1572-82.

4. Oeffinger KC, Nathan PC, Kremer LC. Challenges after curative treatment for childhood cancer and long-term follow up of survivors. Pediatr Clin North Am 2008;55:251-73.

5. Children's Oncology Group. Long-term follow-up guidelines for survivors of childhood, adolescent, and young adult cancers, version 2.0. 2006. Available at: http://www.survivorshipguidelines.org. Accessed 17 October 2008.

6. Kadan-Lottick NS, Robison LL, Gurney JG, et al. Childhood cancer survivors' knowledge about their past diagnosis and treatment: childhood cancer survivor study. JAMA 2002;287:1832-9.

7. Nathan PC, Greenberg ML, Ness KK, et al. Medical care in long-term survivors of childhood cancer: a report from the Childhood Cancer Survivor Study (CCSS). J Clin Oncol 2008;26:4401-9.

8. Oeffinger KC. Childhood cancer survivors and primary care physicians. J Fam Pract 2000;49:68990.

9. Cheung WY, Neville AB, Cameron DB, Cook EF, Earle CC. Comparisons of patient and physician expectations for cancer survivorship care. J Clin Oncol 2009;27:2489-95.

10. Friedman DL, Freyer DR, Levitt GA. Models of care for survivors of childhood cancer. Pediatr Blood Cancer 2006;46:159-68.

11. Schulman LN, Jacobs LA, Greenfield S, et al. Cancer care and cancer survivorship care in the United States: will we be able to care for these patients in the future? J Oncol Pract 2009;5(3):119-12.

12. Oeffinger KC. Longitudinal risk-based health care for adult survivors of childhood cancer. Curr Probl Cancer 2003;27:143-67.

13. Hudson MM, Mertens AC, Yasui Y, et al. Health status of adult long-term survivors of childhood cancer: a report from the Childhood Cancer Survivor Study. JAMA 2003;290:1583-92.

14. Park MJ, Mulye TP, Adams SH, Brindis CD, Irwin CE. The health status of young adults in the united states. J Adolesc Health 2006;39:305-17.

15. Werba BE, Hobbie W, Kazak AE, Ittenbach RF, Reilly AF, Meadows AT. Classifying the intensity of pediatric cancer treatment protocols: the intensity of treatment rating scale 2.0 (ITR-2). Pediatr Blood Cancer 2007;48:673-7.

16. Pai AL, Patino-Fernandez AM, McSherry M, et al. The psychosocial assessment tool (PAT 2.0): psychometric properties of a screener for psychosocial distress in families of children newly diagnosed with cancer. J Pediatr Psychol 2008;33:50-62.

17. Lown EA, Goldsby R, Mertens AC, et al. Alcohol consumption patterns and risk factors among childhood cancer survivors compared to siblings and general population peers. Addiction $2008 ; 103$ : $1139-48$.

18. Alderfer MA, Long KA, Lown EA, et al. Psychosocial adjustment of siblings of children with cancer: a systematic review. Psychooncology 2009; Epub ahead of print.

19. Mao JJ, Armstrong K, Bowman MA, Xie SX, Kadakia R, Farrar JT. Symptom burden among cancer 
survivors: impact of age and comorbidity. J Am Board Fam Med 2007;20:434-43.

20. Laakso V, Niemi PM, Grönroos M, Aalto S, Karlsson $\mathrm{H}$. The worried young adult as a primary care patient. Fam Pract 2005;22:406-11.

21. Mao JJ, Bowman MA, Stricker C, at al. Delivery of survivorship care by primary care physicians: the perspective of breast cancer patients. J Clin Oncol 2009;27:933-8.

22. Gurney JG, Kadan-Lottick NS, Ness KK, Bhatia S.
Survival variability by race and ethnicity in childhood acute lymphoblastic leukemia. JAMA 2003; 290:2008-14.

23. Robison LL, Mertens AC, Boice JD, et al. Study design and cohort characteristics of the Childhood Cancer Survivor Study: a multi-institutional collaborative project. Med Pediatr Oncol 2002;38:229-39.

24. Oeffinger KC, Robison LL. Childhood cancer survivors, late effects, and a new paradigm for understanding survivorship. JAMA 2007;297:2762-4. 